\title{
ROOF STRUCTURE OF CLOSE DISTANCE COAL STRATA IN MULTI-GOB CONDITION AND ITS EFFECTS
}

\author{
Jingxuan X. YANG ${ }^{1)}$, Changyou Y. LIU ${ }^{1) *}$, Bin YU ${ }^{2)}$ and Fengfeng F. WU ${ }^{1)}$ \\ ${ }^{1)}$ School of Mines, State Key Laboratory of Coal Resources and Safe Mining, Key Laboratory of Deep Coal Resource Ming Ministry of \\ Education, China University of Mining \& Technology, Xuzhou, Jiangsu 221116, China \\ ${ }^{2}$ Datong Coal Mine Group Company, Datong, Shanxi 037003, China \\ *Corresponding author's e-mail: cyliucumt@163.com
}

\section{ARTICLE INFO}

\section{Article history}

Received 28 June 2014

Accepted 30 October 2014

Available online 24 November

Keywords:

Close distance strata

Multi-gob

Roof structure

Stress of coal pillars

Natural arch

\section{ABSTRACT}

To ensure the safety of excavation of carbonic coal strata in Datong minefields, the effect of multi-gob roof structure after the excavation of upper Jurassic close distance coal strata needs to be analysed. The multi-gob roof structure of Jurassic close distance and its effect was evaluated by theoretical analysis in this study. The types of multi-gob roof structure of close distance coal strata were categorised and the stress distribution in each type of structure was analysed. Results showed that: the blocks of the upper roofing of the gobs of Jurassic coal strata in the inclined direction of the working face extrude with each other, resulting in stable natural arch structure. The arch springing acts on the coal pillars in the gobs, resulting in significant concentrated load; according to the location of reservation of coal pillars in the gobs and spacing of coal strata, the roof structure of the gobs of close distance coal strata can be categorised into four types. Calculating equations of the load of coal pillars and waste rocks in the gobs for each type of structure were proposed; with respect to the condition of production of upper Jurassic coal strata in Tongxin coal mines, the multi-gob roofing of the coal strata of the mine was determined as aligned coupled structure and the effective depth of coal pillars in the gobs of the respective roof structure was calculated. Results from this study provide basis for the prediction and forecast of the mine pressure on the working face of underneath carbonic coal strata as well as the analysis of pressure strength.

\section{INTRODUCTION}

There are abundant coal resources in Datong minefields in Shanxi Province in China. Affected by room and pillar mining and small coal pit mining at the early stage and due to the requirement to protect the adjacent working face, a large amount of coal pillars were setup in the gobs of coal strata. Due to the excavation of close distance coal strata and the effect of coal pillars in the gobs, the roof structure of the gobs of excavated Jurassic coal strata is relatively complex. At present, the mining of Jurassic coal strata in the mine fields in Datong has almost completed and the carbonic thick and extra thick coal strata become the primary coal strata for mining. To ensure the safety of excavation of lower carbonic coal strata, it is necessary to analyse the multi-gob roof structure and its effect of excavated upper Jurassic coal strata. It is known that when the coal stratum is excavated, the immediate roof stratum collapses naturally and fills the space of the gob. For upper hard rock-overlaid roof strata, masonry beam structure (Qian et al., 1994) is formed when the strata collapses into blocks. The stability of such structure is maintained by the extrusive action among the collapsed blocks so that the self-weight and the weight of rock-overlaid strata can be sustained. Natural arch structure is formed among the overlying strata due to horizontal extrusive force among the collapsed blocks from the roof strata.
A large number of sectional coal pillars were setup in the adjacent working faces during the excavation of Jurassic coal strata, the self-weight of the rockoverlaid natural arch structure are sustained by the coal pillars when the coal strata were excavated. As a result, the coal pillars in the gob are subjected to concentrated force from natural arch roof structure which effects lower strata to a certain extent ( $\mathrm{Yu}$ et al., 2014; Wang and Hou., 2003; Yang et al., 2013).

At present, significant amounts of research were conducted on the analysis of natural arch structure: with respect of the stability issues of tunnel, Miu et al. (1990) established natural arch structural model from the prospect of theoretical analysis and acquired the range of influence of the excavation of the enclosing rocks of the tunnel; Wang et al. (2007) analysed the collapse mechanism of ground surface under shallowburied hard overlying rocks by numerical simulation and proposed the concept of combined stress arch of the stope. The self-weight of the overlying rocks was applied ultimately on the coal pillars in the gobs by combined stress arch structure; Du et al. (2011) acquired the evolutionary characteristics of the pressure arch of the stope by numerical analysis and resemblance simulation from the perspective that the roof structure of the working face is loaded and pressure arch is formed as a result; Wang et al. (Wang and He, 2006) analysed the stability issue of chamber 
Table 1 Parameters of dual-system coal rocks in Tongxin mines.

\begin{tabular}{|c|c|c|c|c|c|c|c|c|c|}
\hline No. & $\begin{array}{l}\text { Lithological } \\
\text { characteristics }\end{array}$ & $\begin{array}{c}\text { Thickness } \\
\text { m }\end{array}$ & $\begin{array}{l}\text { Density } \\
\mathrm{kg} / \mathrm{m}^{3}\end{array}$ & $\begin{array}{l}\text { Compressive } \\
\text { strength } \\
\mathrm{MPa}\end{array}$ & $\begin{array}{c}\text { Tensile } \\
\text { strength } \\
\mathrm{MPa}\end{array}$ & $\begin{array}{l}\text { Elastic } \\
\text { modulus } \\
\mathrm{GPa}\end{array}$ & $\begin{array}{l}\text { Cohesion } \\
\mathrm{MPa}\end{array}$ & $\begin{array}{l}\text { Internal } \\
\text { angle of } \\
\text { friction }\end{array}$ & $\begin{array}{l}\text { Poisson's } \\
\text { ratio }\end{array}$ \\
\hline 1 & packsands & 15 & 2595 & 75.5 & 10.8 & 38.1 & 18.4 & 47 & 0.10 \\
\hline 2 & medium gritstone & 6.2 & 2534 & 59.3 & 10.5 & 21.5 & 10.2 & 31 & 0.17 \\
\hline 3 & sandy mudstone & 4.3 & 2595 & 63.8 & 7.8 & 35.1 & 8.3 & 33 & 0.22 \\
\hline 4 & $11 \#$ coal & 4.3 & 1426 & 24.8 & 3.9 & 4.2 & 14.3 & 30 & 0.32 \\
\hline 5 & packsands & 11.0 & 2595 & 75.5 & 10.8 & 38.1 & 18.9 & 47 & 0.10 \\
\hline 6 & medium gritstone & 7.1 & 2534 & 59.3 & 10.5 & 21.5 & 10.2 & 31 & 0.17 \\
\hline 7 & sandy mudstone & 3.3 & 2595 & 63.8 & 7.8 & 35.1 & 8.3 & 33 & 0.22 \\
\hline 8 & $12 \#$ coal & 2.5 & 1426 & 24.8 & 3.9 & 4.2 & 14.3 & 30 & 0.32 \\
\hline 9 & packsands & 6.8 & 2595 & 75.5 & 10.8 & 38.1 & 23.6 & 47 & 0.10 \\
\hline 10 & $14 \#$ coal & 2.1 & 1426 & 24.8 & 3.9 & 4.2 & 14.3 & 30 & 0.32 \\
\hline 11 & packsands & 14.1 & 2595 & 75.5 & 10.8 & 38.1 & 20.6 & 47 & 0.10 \\
\hline 12 & sandy mudstone & 1.7 & 2595 & 63.8 & 7.8 & 35.1 & 8.3 & 33 & 0.22 \\
\hline 13 & $15 \#$ coal & 4.2 & 1426 & 24.8 & 3.9 & 4.2 & 14.3 & 30 & 0.32 \\
\hline 14 & packsands & 10.3 & 2595 & 75.5 & 10.8 & 38.1 & 19.4 & 47 & 0.10 \\
\hline 15 & medium gritstone & 10.0 & 2534 & 59.3 & 10.5 & 21.5 & 10.2 & 31 & 0.17 \\
\hline 16 & medium siltstone & 106.5 & 2534 & 59.3 & 10.5 & 27.5 & 14.4 & 37 & 0.24 \\
\hline 17 & medium gritstone & 10.8 & 2534 & 59.3 & 10.5 & 21.5 & 10.2 & 31 & 0.17 \\
\hline 18 & sandy mudstone & 55.0 & 2595 & 63.8 & 7.8 & 35.1 & 8.3 & 33 & 0.22 \\
\hline 19 & ${ }_{4}^{\#}$ coal & 2.4 & 1426 & 24.8 & 3.9 & 4.2 & 14.3 & 30 & 0.32 \\
\hline 20 & $\begin{array}{l}\text { mudstone and } \\
\text { siltstone } \\
\text { interbedding }\end{array}$ & 14.4 & 2747 & 54.6 & 7.5 & 27.9 & 8.3 & 29 & 0.18 \\
\hline 21 & sandstone & 2.2 & 2747 & 60.2 & 8.4 & 35.4 & 12.8 & 30 & 0.18 \\
\hline 22 & magmatite & 1.7 & 2747 & 106.8 & 16.1 & 50.9 & 18.7 & 50 & 0.10 \\
\hline 23 & 2\# coal & 4.2 & 1426 & 24.8 & 3.9 & 4.2 & 14.3 & 30 & 0.32 \\
\hline 24 & carbon mudstone & 2.9 & 2728 & 39.6 & 6.0 & 35.1 & 8.3 & 33 & 0.22 \\
\hline 25 & petrosilex & 1.7 & 2595 & 113.1 & 13.4 & 53.9 & 20.6 & 50 & 0.10 \\
\hline 26 & $3-5 \#$ coal & 14.1 & 1426 & 24.8 & 3.9 & 4.2 & 14.3 & 30 & 0.32 \\
\hline 27 & Kaolin & 5.0 & 2595 & 63.8 & 7.8 & 35.4 & 12.8 & 30 & 0.18 \\
\hline
\end{tabular}

and proposed the equations of the height of limit equilibrium for chambers by Protodyakonov's theory of natural arch. However, only single rock enclosing condition was considered in most studies and the analysis of roof structure under multi-gob condition of coal strata and the effect of coal pillars was rare. Therefore, on the background of the mining conditions of Jurassic coal strata in Datong mine fields, this study analysed the roof structure of the gobs of Jurassic close distance coal strata and its effect by theoretical analysis; the structural characteristics of the fractured roof structure of the gobs of excavated Jurassic coal strata and the range of influence were evaluated. Results from this study provides basis for the prediction and forecast of the mine pressure on the working face of underneath carbonic coal strata as well as the analysis of pressure strength.

\section{OCCURRENCE OF COAL STRATA AND CHARACTERISTICS OF EXCAVATION}

Both Jurassic and carbonic coal strata exist in Tongxin mines in Datong minefields, Shangxi Province. Take the excavation of the dual-system coal strata at the No.1 panel at the northern part of the mine as an example, the average length in the northsouth and west-east direction at the panel were $6.7 \mathrm{~km}$ and $4.0 \mathrm{~km}$, respectively. The carbonic coal strata in the panel was stoped directly through the main tunnel of the No.1 panel at the north. One-way excavation 




Fig. 1 Layout of the working faces of the dual-system coal strata in Tongxin mines.

was conducted on the stoping tunnels positioned perpendicular to the main tunnel according to the shape of the panel. The excavation of the upper Jurassic coal strata was completed in 1970 s to 1980 s, in which the working faces of coal strata in 1980s were positioned perpendicular to the direction of excavation of the working faces of underneath carbonic coal strata while in 1970 s, the dimensions of the working faces of the coal strata were relatively small due to the limitation of excavating techniques and the direction of those working faces was basically parallel to the direction of excavation of underneath carbonic coal strata.

Presently, the upper parts of carbonic coal strata at Tongxin mines in Datong minefields correspond to the major part of the excavated area of Jurassic 11\#, 12\# and 14\# coal strata. The distance of Jurassic and carbonic coal strata is approximately 150 to $200 \mathrm{~m}$ which is filled with hard rock strata include packsands, siltstone, medium grained sand and conglomerate. In particular, sandy rock strata take up $90 \%$ to $95 \%$ while muddy rock and coal strata take up only $5 \%$ to $10 \%$. The parameters of the dualsystem coal strata and layout of the working faces of the coal strata are shown in Table 1 and Figure 1, respectively.

The slope angle of the dual-system coal strata in Tongxin mines is small with an average of 1 to $3^{\circ}$, which can be categorised as flat coal strata. Multiple coal strata exist in the upper part of the mines. Coal strata with a spacing of 5 to $22 \mathrm{~m}$ are classified as close distance coal strata; those with thicknesses of 2.0 to $4.5 \mathrm{~m}$ are medium thick coal strata; the lower carbonic coal strata 3-5\# which are currently excavating have a larger thickness with an average value of $14.1 \mathrm{~m}$. Take the production of the coal mines in Tongxin coal mine as an example, for Working Face 8104 to 8107 of carbonic coal strata which are currently excavated, gobs of the two Jurassic close distance coal strata and sectional coal pillars reserved in the adjacent working faces were distributed on the upper part.

Due the excavation of upper Jurassic coal strata at the early stage and large gobs of the lower carbonic coal strata, during the production process of lower extra-thick coal strata, the perturbation and excavation of the working faces may once again affect the stability of roof structure of the gobs of overlying Jurassic excavated coal strata. At this stage, the activated roof structure may affect the normal excavation process of underneath carbonic coal strata. Therefore, in order to ensure the normal excavation of the carbonic extra-thick coal strata, it is necessary to analyse the roof structure of the upper Jurassic earlystage excavated coal strata and its effects.

Due to the fact that the direction of layout of the working faces of Jurassic coal strata is different and affected by the excavation of close distance coal strata and coal pillars, the multi-gob roof natural arch structure is complex and natural arch structure is formed between the roofing of adjacent working faces. According to the difference of the direction of layout of the working faces of Jurassic coal strata at Tongxin coal mine and the location of coal pillars in the gobs (as shown in Figure 1), the combined natural arch structure of the roof of the gobs of close distance coal strata can be categorised into aligned and misaligned types, as shown in Figure 2. 


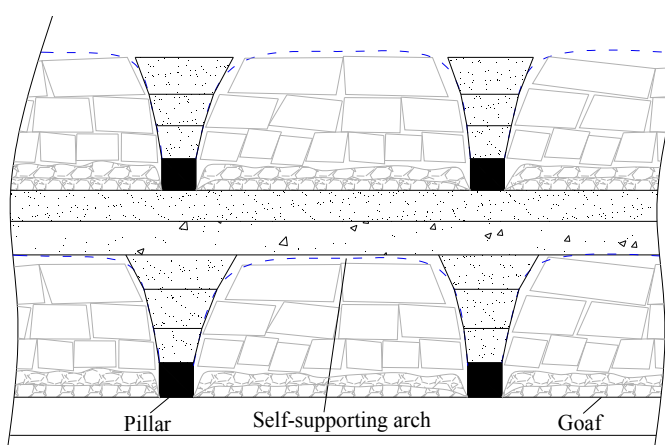

(a) Aligned structure

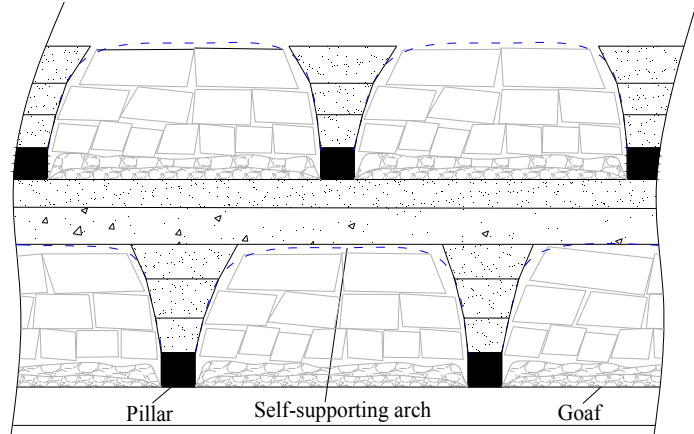

(b) Misaligned structure

Fig. 2 Natural arch structure of the roofing of close distance coal strata.

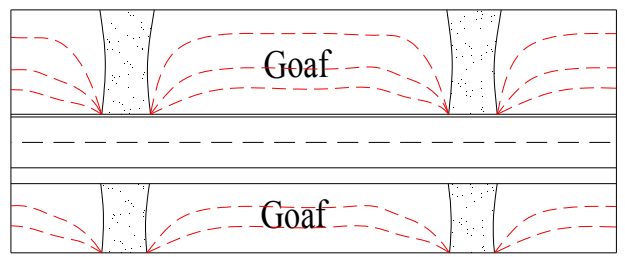

(a) Aligned independent structure

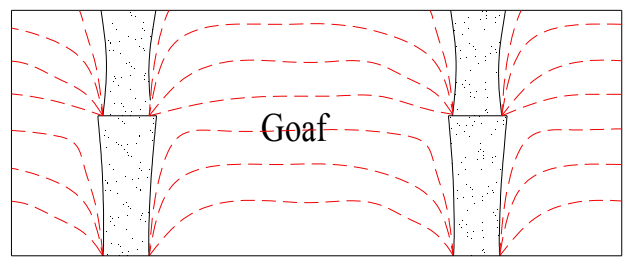

(c) Aligned coupled structure

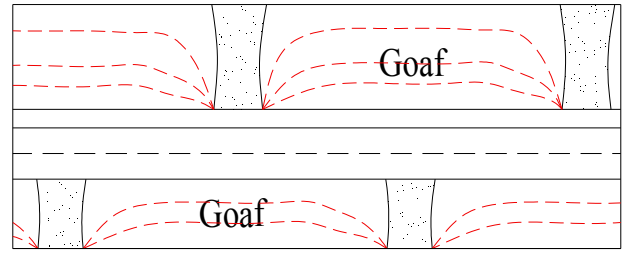

(b) Misaligned independent structure

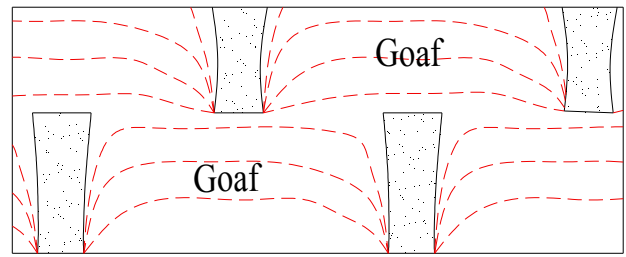

(d) Misaligned coupled structure

Fig. 3 Schematic diagram for the categorisation of combined natural arch structure.

Meanwhile, according to the difference of spacing of close distance coal strata, each type of combined natural arch structure analysed above can be further categorised into independent and coupled structures, as shown in Figure 3.

It is evident from Figures 2 and 3 that the selfweight of overlying rocks after the excavation of close distance coal strata is applied ultimately on the lower coal stratum flooring via coal pillars and the waste rocks in the gobs. Therefore, the stress distribution in the combined natural arch structure of rock-overlaid roofing of close distance coal strata is evaluated in this study to provide basis for the analysis of the effect of concentrated stress on the coal pillars in the gobs of Jurassic coal strata.

\section{THEORETICAL STUDY}

\subsection{ANALYSIS OF NATURAL ARCH STRUCTURE OF SINGLE ROOFING}

In order to analyse the load-carrying characteristics of the combined natural arch structure of multi-gob roofing of Jurassic close distance coal strata in Datong minefields, the natural arch structure of the gobs after the excavation of a single working face of coal strata is analysed first. The natural arch theory is introduced to analyse the formation of natural arch structure of the roofing of the gobs of working faces. The geometric characteristics of the natural arch of single roofing of the gob is demonstrated in Figure 4.

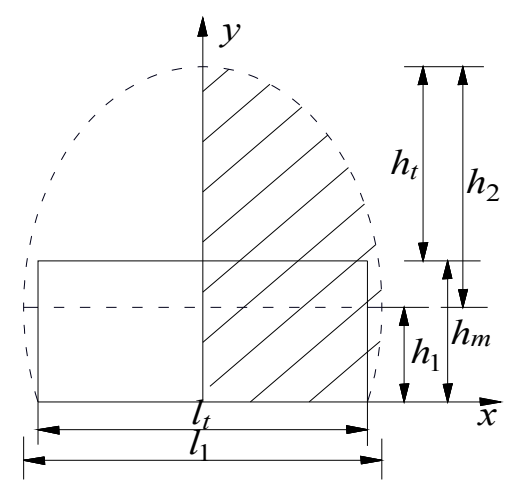

Fig. 4 Geometric characteristics of natural arch. 
The geometric shape of the natural arch structure of the roofing of the gobs is generally elliptical (Miao, 1990; Wang et al., 2007; Du et al., 2011; Wang and $\mathrm{He}, 2006)$. Therefore, the equation of the natural arch of the roofing of the gobs is assumed as:

$\frac{4 x^{2}}{l_{1}^{2}}+\frac{\left(y-h_{1}\right)^{2}}{h_{2}^{2}}=1$

In which: $l_{1}$ is the width of the natural arch; $h_{1}$ is the distance from the centre of the natural arch to the coal stratum flooring; $h_{2}$ is the height of the natural arch.

According to the mechanical equilibrium of the natural arch structure, $\mathrm{t}$ the width and height of the natural arch satisfies the following relationship (Miao, 1990):

$l_{1}^{2}=4 \eta h_{2}^{2}$

In which $\eta$ is the coefficient of lateral pressure.

Incorporate Equations 1 and 2, the geometric relationship of the natural arch structure of single roofing is given as:

$$
\left\{\begin{array}{c}
H=h_{t}+h_{m}=d+\sqrt{\frac{l_{t}^{2}+4 d^{2}}{4 \eta}} \\
h_{1}=\frac{H}{2}-\frac{l_{t}^{2}}{8 H \eta}, \quad h_{2}=\frac{H}{2}+\frac{l_{t}^{2}}{8 H \eta} \\
l_{1}=H \sqrt{\eta}+\frac{l_{t}^{2}}{4 H \sqrt{\eta}}
\end{array}\right.
$$

where

$$
d=\frac{l_{t} \tan \phi+h_{m}\left(\eta+\tan ^{2} \phi\right)}{2 \eta}, \quad \phi=\frac{\pi}{4}-\frac{\varphi}{2}
$$

In which: $H$ is the distance from the top of natural arch to the coal stratum flooring; $h_{m}$ is the thickness of the coal stratum; $l_{t}$ is the inclined length of the working face; $h_{t}$ is the collapsed height of the gob; $d$ is an intermediate variable; $\varphi$ is the internal angle of friction of the rock; $\phi$ is the collapsing angle of the coal stratum.

In order to calculate the self-weight of the upper roof structure on the coal pillars in the gobs, the shaded area in Figure 4 can be calculated as:

$$
S=\frac{l_{1} h_{2}}{4}\left\{\vartheta_{2}-\vartheta_{1}+\frac{1}{2}\left[\sin \left(2 \vartheta_{2}\right)-\sin \left(2 \vartheta_{1}\right)\right]\right\}
$$

where

$$
\vartheta_{1}=\arcsin \left[\frac{l_{t}^{2}-4 \eta H^{2}}{l_{t}^{2}+4 \eta H^{2}}\right], \quad \vartheta_{2}=\pi / 2
$$

In which $S$ is the area of the shaded parts in Figure 4.

According to the principle of area complementation (as shown in Figure 5), the selfweight of the upper roof structure on a single coal pillars in the gobs can be calculated as:
$Q_{d}=\gamma\left[H\left(l_{m}+l_{t}\right)-2 S\right]$

In which: $Q_{d}$ is the self-weight of the upper roof structure on a single coal pillars; $\gamma$ is the average volumetric weight of the overlying rocks; $l_{m}$ is the width of the coal pillar.

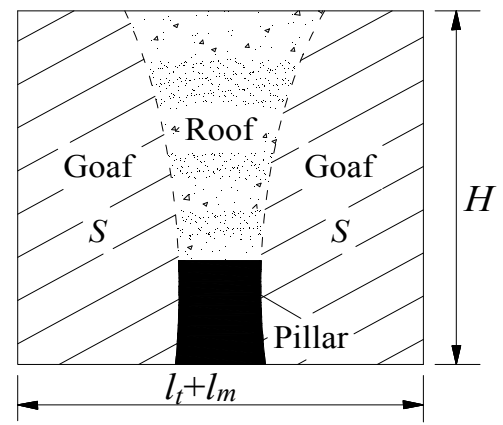

Fig. 5 Upper roof structure on a single coal pillar.

\subsection{ANALYSE OF COMBINED NATURAL ARCH} STRUCTURE OF MULTI-GOBS

\subsubsection{DETERMINATION OF COMBINED NATURAL ARCH STRUCTURE}

As the geometric dimensions and technics are similar for adjacent working faces of the same coal stratum, the length of working faces and width of coal pillars in the coal stratum are basically identical. Therefore, the rock-overlaid roof structure in different gobs are similar to a certain extent.

The conditions of determination of combined natural arch structure of multi-gob roofing in the condition of excavation of close distance coal strata are given as:

$\left\{\begin{array}{l}\text { aligned } \begin{cases}\text { independent structure } & H<h_{d} \\ \text { coupled structure } & H \geqslant h_{d}\end{cases} \\ \text { misaligned } \begin{cases}\text { independent structure } & H<h_{d} \\ \text { coupled structure } & H \geqslant h_{d}\end{cases} \end{array}\right.$

In which $h_{d}$ is the spacing of close distance coal strata.

It is evident from Eq. 6 that if the spacing of close distance coal strata is less than the height of natural arch due to the excavation of lower coal stratum, the roofing of the gobs will penetrate each other when the two coal strata are excavated. In the case, the natural arch structure of the gobs of close distance coal strata are coupled; conversely, due to the spacing of the complete stratum, the natural arch structure in the gobs of two strata are independent to each other.

\subsubsection{FORCE ANALYSIS OF LOWER COAL STRATUM FLOORING}

(1) Aligned independent natural arch structure of close distance coal strata

The inclined length of the working faces of the coal strata is relatively large, the arrangement of waste rocks under the natural arch in the gobs is loose and 
the horizontal force in the waste rocks is small. According the state of natural arch structure of roofing shown in Figure $3 \mathrm{a}$, the force analytical model of multi-gobs coal rocks in close distance coal strata is shown in Figure 6.

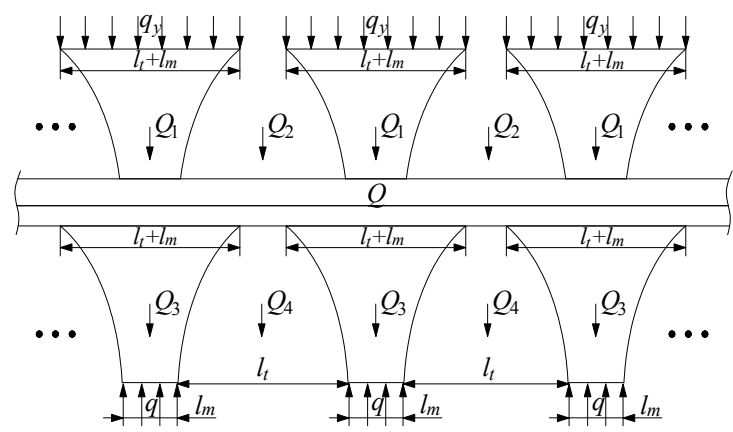

Fig. 6 Force analysis of aligned independent natural arch.

It is evident from Figure 6 that the due to the spacing of complete stratum in close distance coal strata, the weight of the natural arch of upper coal stratum, waste rocks in the gobs and the complete rock stratum between the two coal strata is fully sustained by the natural arch of lower coal stratum, but the weight of waste rocks in the gobs of lower coal stratum acts independently on the coal stratum flooring. Therefore, according to the symmetry properties of overlying rock structure and vertical equilibrium of roof structure, it holds that:

$$
q_{y}\left(l_{m}+l_{t}\right)+Q_{1}+Q_{2}+Q_{3}+\frac{Q}{n-1}=q l_{m}
$$

In which : $q_{y}$ is the force of overlying rocks on the roofing of upper coal stratum; $Q_{1}$ and $Q_{3}$ are respectively the weight of natural arch springing for each coal stratum; $Q_{2}$ is the weight of waste rocks in the gobs of upper coal stratum; $Q$ is the weight of complete rock stratum between the two coal strata in the range of excavation of close distance coal strata; $n$ is the number of working faces in the range of excavation of coal strata; $q$ is the load of lower coal stratum flooring on the natural arch springing.

According to the geometric characteristics of roof structure of close distance coal strata (as shown in Figure 6) and Equation 5, it can be calculated that:

$$
\left\{\begin{array}{c}
Q_{2}=2 \gamma S_{1} \\
Q_{1}+Q_{2}=\gamma H_{1}\left(l_{m}+l_{t}\right) \\
Q=\gamma\left[n l_{t}+(n-1) l_{m}\right] h_{c} \\
Q_{3}=\gamma\left[H_{2}\left(l_{m}+l_{t}\right)-2 S_{2}\right]
\end{array}\right.
$$

In which: $H_{1}$ and $H_{2}$ are distance from the top of natural arch to the coal stratum flooring for each coal stratum; $S_{1}$ and $S_{2}$ are the area corresponds to the natural arch of each coal stratum; $h_{c}$ is the thickness of complete rock stratum between the two coal strata.
Incorporate Equations 7 and 8, the load of lower coal stratum flooring on the coal pillars in the gobs can be calculated as:

$$
q=\left[\left(q_{y}+\gamma H_{z}\right)\left(l_{m}+l_{t}\right)-2 \gamma S_{2}+\frac{\gamma h_{c} l_{t}}{n-1}\right] / l_{m}
$$

In which $H_{z}=H_{1}+H_{2}+h_{c}=H_{1}+h_{d}$.

The weight of waste rocks in the natural arch of the gobs of lower coal stratum acts directly on the coal stratum flooring. Therefore, the average load of the flooring of the gobs of lower coal stratum can be calculated as:

$$
q_{c}=Q_{4} / l_{t}=\gamma\left[H_{2}\left(l_{m}+l_{t}\right)-2 S_{2}\right] / l_{t}
$$

In which $q_{c}$ is the average load of the flooring of the gobs of lower coal stratum.

\section{(2) Misaligned independent natural arch} structure of close distance coal strata

For misaligned independent natural arch structure of close distance coal strata, the loading condition of the natural arch of the gobs of lower coal stratum is similar to the case of aligned independent structure. Due to the spacing of complete stratum in close distance coal strata, the weight of the natural arch of upper coal stratum, waste rocks in the gobs and the complete rock stratum between the two coal strata is also fully sustained by the natural arch of lower coal stratum. In this case, the force analytical model of the natural arch of lower close distance coal strata is shown in Figure 7.

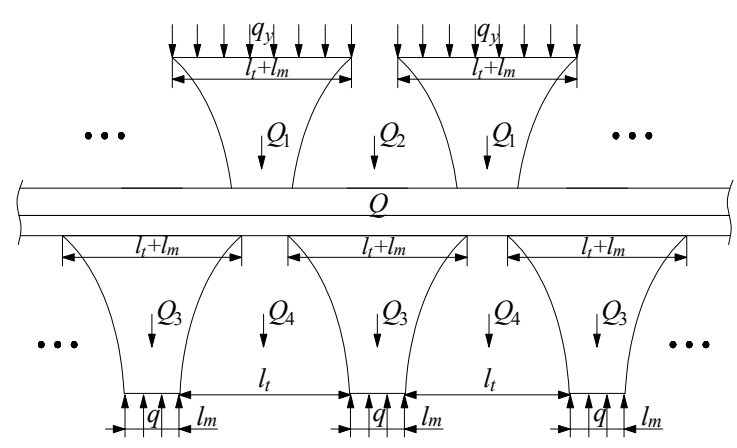

Fig. 7 Force analysis of misaligned independent natural arch.

It is evident from Figure 7 that in the condition of multi-gob misaligned independent natural arch structure, a certain level of symmetric properties exists in the roof structure of coal strata. Moreover, affected by the complete rock stratum in between, the overlying rock loading sustained by the natural arch of lower coal stratum is basically identical. Therefore, in the condition of identical excavating range of coal strata, the average load of coal pillars and flooring in the gobs of lower coal stratum can also be calculated by respectively Equations 9 and 10 . 




Fig. 8 Force analysis of aligned coupled natural arch

(3) Aligned coupled natural arch structure of close distance coal strata

When the height of natural arch after the excavation of lower coal stratum is larger than the spacing of coal strata, the roof structure of the two coal strata couples with each other. As the compressive ratio of waste rocks in the gobs is relatively large, the weight of overlying rocks in the gobs will be sustained by natural arch of both coal strata, while the weight of waste rocks of the two coal strata applies directly on the bottom as static load. In this case, the force analytical model of aligned coupled natural arch structure in the gobs is shown in Figure 8.

Similarly, according to vertical force equilibrium of natural arch in the gobs, the load of lower coal stratum flooring on the coal pillars in the gobs can be calculated as:

$q=\left[q_{y}\left(l_{m}+l_{t}\right)+Q_{1}+Q_{3}\right] / l_{m}$

It is worth pointing out that due to the spacing of close distance coal strata is small, in the equation of the weight of arch springing of lower coal stratum $Q_{3}$, the height of natural arch $\mathrm{H}_{2}$ should be replaced by the spacing of coal strata $h_{d}$, and the spacing $h_{d}$ can be substitute into Equation 4 to calculate relevant area $S_{2}$.

The loading of flooring of lower coal strata due to the static load of waste rocks of the gobs of the two coal strata can be calculated according to the principle of hydrostatic pressure as:

$q_{c}=\gamma\left(h_{d}+H_{1}\right)$

(4) Misaligned coupled natural arch structure of close distance coal strata

The load analysis of misaligned coupled natural arch structure of close distance coal strata is shown in Figure 9.

According to vertical force equilibrium of natural arch in the gobs, the load of lower coal stratum flooring on the coal pillars in the gobs can be calculated as

$q=\left(2 \gamma S_{1}+Q_{3}\right) / l_{m}$

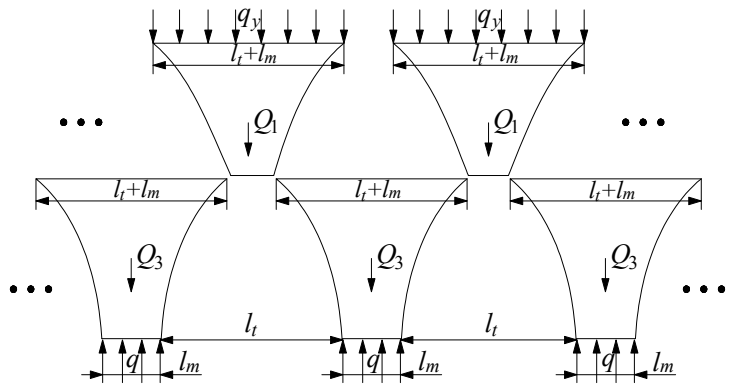

Fig. 9 Force analysis of misaligned coupled natural arch.

Similarly, the load of waste rocks in the gobs of lower coal stratum on the coal stratum flooring can be calculated as:

$q_{c}=\left[q_{y}\left(l_{m}+l_{t}\right)+Q_{1}+2 \gamma S_{2}\right] / l_{t}$

For Equations 13 and 14, the weight of natural arch springing of lower coal stratum and area of the gobs can be calculated similarly to Equation 11 .

In conclusion, the types of natural arch structure of multi-gob roofing and respective equations of coal rock load in the condition of excavation of close distance coal strata have been analysed. Therefore, in the prescribed excavating conditions, the type of roof structure of close distance coal strata should be determined first and the calculating method of coal rock load can be selected accordingly to analyse the effect of respective loading on the lower rock strata.

\section{APPLIED ANALYSIS}

The production of Working Face 8105 of carbonic coal strata in Tongxin coal mine is selected as background. The upper part of the working face is the gobs of two Jurassic close distance coal strata. The average spacing of the close distance coal strata was $15.4 \mathrm{~m}$, the average thickness of the lower and upper coal strata were respectively $3.5 \mathrm{~m}$ and $2.6 \mathrm{~m}$; the inclined length of working faces of the two coal strata was setup as $150 \mathrm{~m}$; the reserved width of coal pillars in the gobs was around $40 \mathrm{~m}$; the average angle of friction of the rock stratum in between was $37^{\circ}$; the Jurassic coal stratum was shallowly buried and the coefficient of lateral pressure of the coal rocks was set as 1 . Therefore, the theoretical value of the height of the natural arch after the excavation of lower Jurassic coal stratum can be calculated by Equation 3 as $121 \mathrm{~m}$, which exceeds the spacing of close distance coal strata. According to the characteristics of working face layout for dual-system coal strata in Figure 1, it can be determined that aligned coupled natural arch structure exists in the multi-gob roofing after the excavation of Jurassic close distance coal strata.

The average burial depth of Jurassic coal strata was around $210 \mathrm{~m}$. Therefore, the load carried by the natural arch of the upper close distance coal stratum can be calculated as $5.4 \mathrm{MPa}$. According to Equations 




Fig. 10 Trapezoidal load of coal pillars.

11 and 12, the load carried by coal pillars in the lower coal stratum and the load applied by waster rocks in the gobs on the coal stratum flooring are calculated as 30.4 MPa and 3.6 MPa, respectively.

Considering that the strength of coal pillars is relatively low and they are not confined, when subjected to high stress imposed by rock-overlaid strata, the coal pillar will undergo complex elastoplastic deformation or damage internally, resulting different load-carrying characteristics in different regions in the coal pillar (Liu et al., 2007; Xie et al., 2006). Normally, the coal pillar is in biaxial loading state at the side closer to the gob in which case plastic deformation or local damage is likely to occur and the load-carrying capacity is relatively low; the stress in the coal body transfers to the inner side of the coal pillar from the edge of coal pillar to the inside, the uniaxial stress state of the coal body shifts gradually to tri-axial stress state and the load-carrying capacity is improved; In the elastic core region of the coal pillar, the boundary effect of the coal pillar is relatively small and the load-carrying capacity of coal body in that region is stable. Based on the aforementioned characteristics of loading and deformation, the load in the established coal pillars in the gobs is trapezoidal load, as shown in Figure 10.

According to the trapezoidal load of the coal pillars in the gobs and the principle of equivalent distributed load analysed previously, in conjunction with the geometric relationship shown in Figure 10, it is clear that:

$$
l_{p}+2 l_{s}=l_{m}, \quad q_{m}\left(l_{s}+l_{p}\right)=q l_{m}
$$

In which $q_{m}$ is the trapezoidal load of coal pillars, $l_{p}$ is the length of elastic core of the coal pillars and $l_{s}$ is the length of stress reduction region of coal pillars.

Therefore, the trapezoidal load of coal pillars in the gobs can be calculated as:

$q_{m}=\frac{2 q l_{m}}{l_{m}+l_{p}}$

After the excavation of Jurassic coal strata in Tongxin coal mine, the waste rocks in the gobs and roofing natural arch structure are in a stable and balanced state through prolong period of kinetic adjustment. In this stage, the natural arch in the gobs possesses a certain level of load-carrying capacity and the waste rocks in the gobs act directly on the coal stratum flooring in the form of self-weight. With respect to the condition of excavation of Jurassic coal strata in Tongxin coal minefields, the force analysis model of rock stratum flooring of the gobs is established as shown Figure 11.

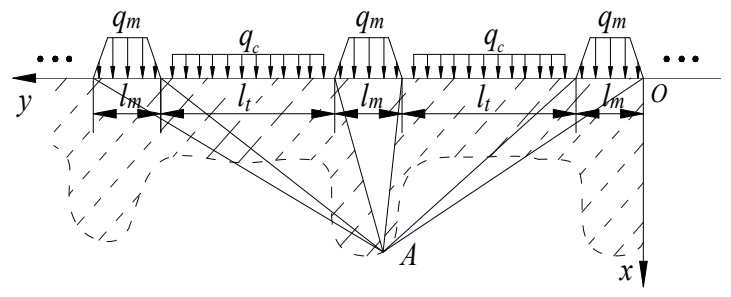

Fig. 11 Force analysis of rock stratum flooring of the gobs.

According to the measured results from the Jurassic coal strata in Datong minefields, the length of stress reduction region at both sides of coal pillars was $5 \mathrm{~m}$ and the length of elastic core of coal pillars was $30 \mathrm{~m}$ (Yang et al., 2014). With respect that sandy stratum lies between the dual-system coal strata in Datong minefields and the lithological characteristics of the roofing are similar, theory of elastic semiinfinite body is used to analyse the stress in the Jurassic rock stratum flooring (Yang et al., 2014; Yang et al., 2012; Xu, 2006). The stress distribution of rock stratum flooring of the gobs of lower close distance coal stratum is shown in Figure 12.

It can be seen from Figure 12 that the existence of natural arch roof structure of Jurassic coal strata leads to stress concentration in the lower rock stratum underneath the coal pillars in the gobs. Results showed that the effective depth of stress concentration in the lower rock stratum underneath the coal pillars in the gobs of lower close distance coal stratum. Compared with the conditions in the coal strata in Datong minefields where the spacing of dual-system coal strata was 150 to $200 \mathrm{~m}$, the excavation of lower carbonic extra-thick coal strata is not yet affected by the coal pillars in the gobs. However, when the kinetic range of overlying rocks due to the excavation of lower coal strata penetrates the effective region of stress concentration of overlying coal pillars, the elastic energy accumulated in the effective region of stress concentration in the overlying rocks will release. In addition, the roof structure of Jurassic coal strata which was originally stable will be reactivated, resulting in significant effect on the excavation of lower carbonic coal strata (Yu et al., 2014).

\section{CONCLUSIONS}

The blocks of the upper roofing of the gobs of Jurassic coal strata in the inclined direction of the working face extrude with each other, resulting in stable natural arch structure. Take the analysis of the 




(a) Vertical stress distribution in rock strata $(\mathrm{MPa})$

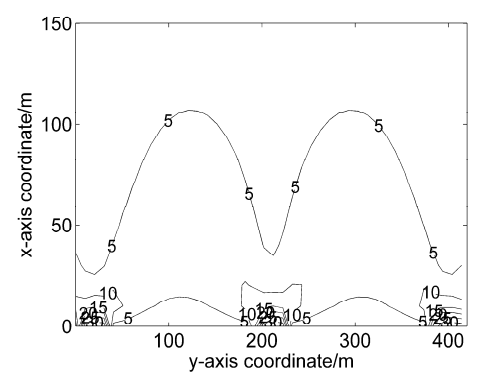

(b) Horizontal stress distribution in rock strata $(\mathrm{MPa})$

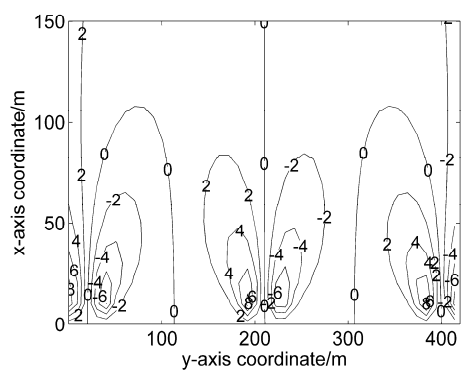

(c) Sheer stress distribution in rock strata $(\mathrm{MPa})$

Fig. 12 Stress distribution of rock stratum flooring of the gobs of lower coal stratum.

natural arch structure of a single roofing as an example, the method of calculation of the weight of natural arch springing of the roofing and the waste rocks in the gobs was proposed in this study according to the loading and geometric characteristics of the roof structure of the gobs.

According to the location of reservation of coal pillars in the gobs and spacing of coal strata, the roof structure of the gobs of close distance coal strata can be categorised into four types: aligned independent structure, misaligned independent structure, aligned coupled structure and misaligned coupled structure. The loading characteristics of coal pillars and waste rocks in the gobs of lower close distance coal stratum were analysed and calculated for each specific type of roofing.

After the excavation of Jurassic close distance coal strata, the loading of coal pillars in the gobs of lower coal strata was relatively concentrated. With a background of production of upper Jurassic close distance coal strata in Tongxin coal mine of Datong minefields, the multi-gob roofing of the coal strata of the mine was determined as aligned coupled structure. The effective depth of stress concentration of coal pillars in the gobs was calculated as around $100 \mathrm{~m}$.

\section{ACKNOWLEDGEMENT}

Financial support for this work, provided by A Special Fund of Major Projects (2014ZDPY21), the Innovation Project of Graduate Students Training of Jiangsu Province (No. CXLX12_0964), and the "333" Training Foundation of Jiangsu Province (No. BRA2010024).

\section{REFERENCES}

Du, X.L., Song, H.W. and Chen, J.: 2011, Numerical simulation of the evolution of the pressure arch during coal mining. Journal of China University of Mining \& Technology, No. 40(6), 863-867, (in Chinese).

Liu, C.Y., Huang, B.X. and Meng, X.J.: 2007, Research on abutment pressure distribution law of over length isolated fully-mechanized top coal caving face. Chinese Journal of Rock Mechanics and Engineering, No. 26 (Supp1), 2671-2676, (in Chinese).
Miao, X.X.: 1990, Natural balance arch and the surrounding rock stability. Ground Pressure and Strata Control, No. (2), 55-57, (in Chinese).

Qian, M.G., Miao, X.X. and He, F.L.: 1994, Analysis of key blocks in the structure of voussoir in longwall mining. Journal of China Coal Society, No.19 (6), 557-563, (in Chinese).

Wang, B. and He, K.Q.:2006, Study on limit equilibrium height expression of critical soil cave of karst collapse. Rock and Soil Mechanics, No. 27(3), 458-462, (in Chinese)

Wang, J.A., Zhao, Z.H. and Hou, Z.Y.: 2007, Study on the catastrophic collapse of surface land induced by mining under a shallow and hard strata. Journal of China Coal Society, No. 32(10), 1051-1056.

Wang, W.J. and Hou, C.J.: 2003, Stability analysis of coal pillar and immediate bottom of extraction opening. Rock and Soil Mechanics, No.24(1), 75-78, (in Chinese).

Xie, G.X., Yang, K. and Liu, Q.M.: 2006, Study on distribution laws of stress in inclined coal pillar for fully-mechanized top-coal caving face. Chinese Journal of Rock Mechanics and Engineering, No. 25(3), 545-549, (in Chinese).

Xu, Z.L.: 2 006, Elastic Mechanics. Beijing: Higher Education Press, (in Chinese).

Yang, J.X., Liu, C.Y. and Yu, B.: 2014, Mechanism of intense strata behaviors at working face influenced by gob pillars of overlying coal seam. Disaster Advances, No. 7(4), 27-35.

Yang, J.X., Liu, C.Y., Yang, Y. and Li, J.W.: 2013, Study of the bearing mechanism of the coal roof and the dimension selection of the room and pillar in the shallow and close distance coal seam. Journal of China University of Mining \& Technology, No. 42(2), 161-168, (in Chinese).

Yang, W., Liu, C.Y., Huang, B.X. and Yang, Y.: 2012, Determination on reasonable malposition of combined mining in close-distance coal seams. Journal of Mining \& Safety Engineering, No. 29(1), 101-105.

Yu, B., Liu, C.Y., Yang, J.X. and Liu, J.R.: 2014, Mechanism of strong pressure reveal under the influence of mining dual system of coal pillar in Datong mining area. Journal of China Coal Society, No. 39(1), 40-46, (in Chinese). DOI: $10.13225 /$ j.cnki.jccs.2013.1482 